\title{
A Logic for Knowledge Flow in Social Networks
}

\author{
Ji Ruan and Michael Thielscher \\ School of Computer Science \& Engineering, The University of New South Wales \\ $\{$ jiruan,mit\}@cse.unsw.edu.au
}

\begin{abstract}
In this paper, we develop a formal framework for analysing the flow of information and knowledge through social networks. Specifically, we propose a multi-agent epistemic logic in which we can represent and reason about communicative actions based on social networks and the resulting knowledge and ignorance of agents. We apply this logic to formally analyse the "Revolt or Stay-at-home" problem known from the literature, where social networks play an important role in agents' knowledge acquisition and decision-making. We evaluate our work by proving some mathematical properties of our new logic, including the fact that it generalises the existing Logic of Public Announcement.
\end{abstract}

\section{Introduction}

The emergence of online social networks such as Facebook and Twitter has enabled richer and easier interactions among people globally. Research on social networks has a long history and is a very interdisciplinary area with important links to sociology, economics, epidemiology, computer science, and mathematics (see $[8,9,1])$. It deals with topics such as exchange of information, spread of diseases, trade of goods and services and diffusion of patterns of social behaviours.

This paper focuses on the modelling of knowledge and ignorance within social networks, and its crucial role in decision-making. As a motivating example, consider the two alternative social networks depicted in Fig. 1, involving four agents, Alice, Bob, Cath and Dave. Suppose they are unhappy about their dictatorial government and consider a revolt. Suppose further that each of them thinks that "I will revolt on condition that I know for sure that at least two others in my social network will also revolt; otherwise I will stay at home." Next, everyone posts their thought to their social network (assuming no government agent is watching and the network structure is common knowledge). Under these assumptions, will these four people actually revolt in these two different social networks? In this scenario, which is an adapted version of an example introduced in [5], the social network structures play a key role in spreading people's intentions and their knowledge about other people's knowledge of their intentions. These intentions can be seen as social interaction protocols, which are specifications for carrying out tasks with specific social goals, such as fair division of desirable goods, rational decision making in groups, voting, and so on.

In this paper, we develop a formal framework for analysing the flow of information and knowledge through social networks. More specifically, we propose 

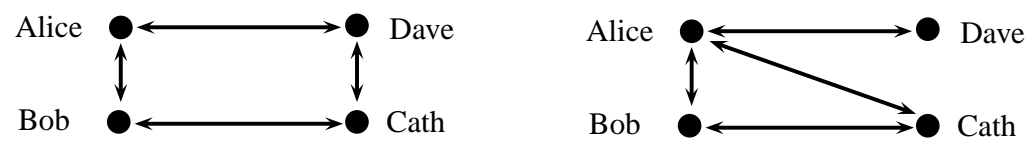

Fig. 1. Two networks structures for the "Revolt or Stay-at-home" scenario.

a multi-agent epistemic logic in which we can represent and reason about communicative actions based on social networks and the resulting knowledge and ignorance of agents.

The remainder of the paper is organised as follows. Section 2 recalls an existing basic version of Dynamic Epistemic Logic: Public Announcement Logic. Section 3 extends this logic with a social network component. The resulting Social Network Logic is then applied to formally analyse the "Revolt or Stay-at-home" problem. In Section 4 we evaluate our new logic by proving some theoretical results. We conclude in Section 5 with a discussion of related work.

\section{Preliminaries}

Dynamic Epistemic Logic [3, 4, 6] studies how actions affect knowledge in a multiagent setting. Public Announcement Logic (PAL) is an example of such a logic and is an extension of standard multi-agent epistemic logic. We give a concise overview of this logic; intuitive explanations of the epistemic part of the semantics can be found in [7].

Definition 1 (The language of Public Announcement Logic). Given are a set of agents $\mathrm{Ag}$ and a set of atoms At. The language of Public Announcement Logic $\mathcal{L}_{\mathrm{PAL}}$ is defined as follows:

$$
\phi \equiv p|\neg \phi| \phi \wedge \psi\left|K_{i} \phi\right| C_{G} \phi \mid\langle\phi\rangle \psi
$$

where $p \in A t, i \in A g, G \subseteq A g$. For $K_{i} \phi$, read 'agent $i$ knows $\phi$.' For $C_{G} \phi$, read ' $\phi$ is common knowledge for the group of agents $G$.' For $\langle\phi\rangle \psi$, read 'after truthful public announcement of $\phi$, formula $\psi$ holds'.

Definition 2 (Epistemic Model). An epistemic model $M$ is a structure $\left\langle W,\left\{\sim_{i}: i \in A g\right\}, V\right\rangle$, where $A g$ is a set of agents, $W$ is a set of possible worlds, each $\sim_{i} \subseteq W \times W$ is an equivalence relation (the accessibility relation) for each agent $i \in A g$, and $V: A t \mapsto 2^{W}$ is a valuation function that assigns each atomic proposition a set of worlds (said to be true in those worlds). For model $M$ and world $w \in W$, entailment is defined as follows:
$M, w \models p \quad$ iff $w \in V(p)$;
$M, w \models \neg \phi \quad$ iff $M, w \not \models \phi$;
$M, w \models \phi \wedge \psi$ iff $M, w \models \phi$ and $M, w \models \psi$;
$M, w=K_{i} \phi \quad$ iff for all $w^{\prime}$, if $w \sim_{i} w^{\prime}$ then $M, w^{\prime} \models \phi$;
$M, w \models C_{G} \phi \quad$ iff for all $w^{\prime}$, if $w \sim_{G} w^{\prime}$ then $M, w^{\prime} \models \phi$;
$M, w \models\langle\phi\rangle \psi \quad$ iff $M, w \models \phi$ and $M \mid \phi, w \models \psi$. 


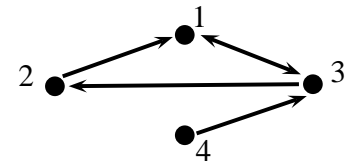

$\mathcal{S}_{1}$

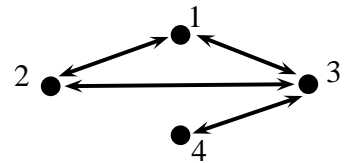

$\mathcal{S}_{2}$

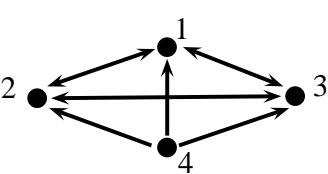

$\mathcal{S}_{3}$

Fig. 2. Example Social Networks.

where the group accessibility relation $\sim_{G}$ is the transitive and reflexive closure of the union of all accessibility relations for the individuals in $G: \sim_{G} \equiv\left(\bigcup_{i \in G} \sim_{i}\right)^{*}$; and the restricted model $M \mid \phi=\left\langle W^{\prime},\left\{\sim_{i}^{\prime}: i \in A g\right\}, V^{\prime}\right\rangle$ is given by

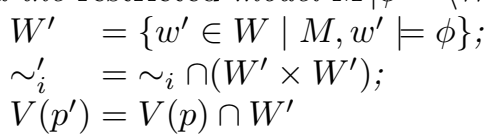

The modal operator $\langle\phi\rangle$ ('after publicly announcing $\phi$ ') is interpreted as an epistemic state transformer: the model $M \mid \phi$ is the model $M$ restricted so as to only contain worlds in which $\phi$ is true. Validity and logical consequence are defined in the standard way. For a proof system, see [6].

\section{Social Network Logic}

In Public Announcement Logic, all agents have the same source of information, making the logic suitable for modelling epistemic problems such as the famous Muddy Children example [7]. However, the language lacks the possibility to explicitly indicate who made the announcement, or to model the case of informing a subgroup of agents with prior dependencies. We address these shortcomings by extending epistemic models with social networks, and the language with more subtle communication actions.

Definition 3 (Social Network). A social network $\mathcal{S}$ is a tuple $\langle A g, F\rangle$, where $A g$ is a set of agents and $F \subseteq A g \times A g \backslash\{(i, i) \mid i \in A g\}$ a binary relation on $\mathrm{Ag}$ (indicating a specific social relation) among agents.

Of all the various types of social relations such as friendship, kinship, common interest, or dislike, we are interested in modelling the relations that influence agents' knowledge acquisition. In particular, a social relation $F$ defines information flow among agents as follows: $i F j$ (or $(i, j) \in F$ ) means that agent $i$ gets information from $j$. Fig. 2 shows three social networks. The second one is symmetric and the third one is transitive. In the following, we will sometimes use the notation $F_{\mathcal{S}}$ to indicate the social relation belonging to social network $\mathcal{S}$.

We extend an epistemic model with a social network component as follows.

Definition 4 (Social Epistemic Model). $A$ social epistemic model $E$ is a structure $\langle A g, \mathcal{S}, M\rangle$, where $A g$ is a set of agents, $\mathcal{S}$ is a social network with $A g$, $M$ is a multi-agent epistemic model with agents $\mathrm{Ag}$. 

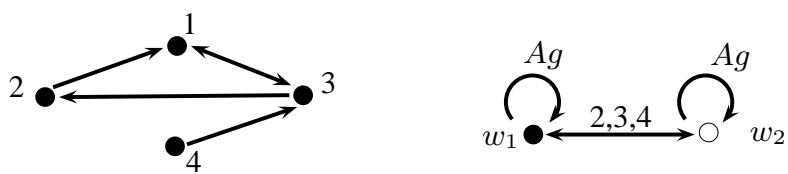

Fig. 3. Example social epistemic mode. Property $p$ is true in $w_{1}$ and false in $w_{2}$.

The common part shared by $\mathcal{S}$ and $M$ is $A g$. In this paper, we assume that $\mathcal{S}$ is common knowledge among $A g$ (see Section 5 for a discussion). Fig. 3 shows a social epistemic model where $A g=\{1,2,3,4\}$, the social network is $\mathcal{S}_{1}$ from Fig. 2, atomic proposition $p$ is true in world $w_{1}$ and false in world $w_{2}$, and only agent 1 can distinguish $w_{1}$ from $w_{2}$ (as indicated by the link between $w_{1}, w_{2}$ being labelled ' $2,3,4$ ', that is, these three agents cannot distinguish $w_{1}$ from $w_{2}$.)

The social network and knowledge of agents can be changed by their actions. We want to capture two kinds of actions:

1. Network Actions: Follow an agent, Unfollow an agent.

Such actions change the social network structure. Suppose $(i, j) \notin F$. If agent $i$ acts to follow agent $j$, then the result will be $(i, j) \in F$. Take, say, $\mathcal{S}_{1}$ from Fig. 2, then executing the three actions '1: Follow 2', '2: Follow 3' and '3: Follow 4' results in $\mathcal{S}_{2}$ in Fig. 2. Action 'Unfollow' has the reverse effect. 2. Message Actions: Post a message $\phi$.

Such actions change agents' knowledge. The effect of agent $i$ posting a message $\phi$ is that all the agents that follow $i$ will know $\phi$. We assume that agents post only messages known to them, that is, both $\phi$ and $K_{i} \phi$ are true.

Definition 5 (Social Epistemic Language). The language of Social Network Logic $\mathcal{L}_{\mathrm{SNL}}$ is defined as follows:

$$
\begin{aligned}
& \phi \equiv p\left|f_{(i, j)}\right| \neg \phi|\phi \wedge \psi| K_{i} \phi\left|C_{G} \phi\right|\langle\pi\rangle \phi \\
& \pi \equiv i: \operatorname{Fo}(j)|i: \operatorname{uFo}(j)| i: \phi
\end{aligned}
$$

where $p \in A t ; f_{(i, j)} \in S A t ; i, j \in A g$; and $G \subseteq A g$.

Unlike the language for Public Announcement Logic, our language has a special kind of atomic propositions $f_{(i, j)}$, indicating that agent $i$ follows $j$, and dynamic modalities $\pi$ for social network changing and message posting. Action ' $i$ : Fo $(j)$ ' means that agent $i$ acts to follow agent $j$; action ' $i: \mathrm{uFo}(j)$ ' means that agent $i$ acts to unfollow $j$; and action ' $i: \phi$ ' means that agent $i$ posts message $\phi$. In terms of the epistemic model, the effect of the last action is to limit all $i$ 's followers' access to worlds in which $\phi$ is true. To define the precise meaning of $i: \phi$, we adapt the concept of action models from Dynamic Epistemic Logic [6] as follows.

Definition 6 (Action Model). Given a social network $\mathcal{S}$, an action model $A_{(k, \phi)}^{\mathcal{S}}$ for agent $k$ posting message $\phi$ in $\mathcal{S}$ is a structure

$$
\left\langle\left\{a_{1}, a_{2}\right\},\left\{\sim_{i}: i \in A g\right\}, p r e\right\rangle
$$




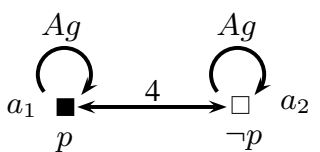

$A_{(1, p)}^{\mathcal{S}_{1}}$

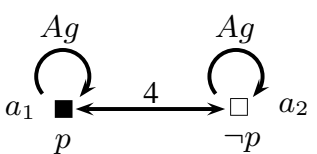

$A_{(1, p)}^{\mathcal{S}_{2}}$
$\overbrace{a_{1}}^{A g}$

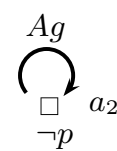

$A_{(1, p)}^{\mathcal{S}_{3}}$

Fig. 4. Example Action Models. $\mathcal{S}_{1}, \mathcal{S}_{2}$ and $\mathcal{S}_{3}$ correspond to those in Fig. 2.

where $a_{1}, a_{2}$ are two atomic actions; pre $\left(a_{1}\right)=\phi$ and pre $\left(a_{2}\right)=\neg \phi$; and $\sim_{i} \subseteq\left\{a_{1}, a_{2}\right\}^{2}$, for which we distinguish two cases: if $i=k$ or $i F_{\mathcal{S}} k$, then $\sim_{i}=\left\{\left(a_{1}, a_{1}\right),\left(a_{2}, a_{2}\right)\right\}$; otherwise, $\sim_{i}=\left\{\left(a_{1}, a_{1}\right),\left(a_{1}, a_{2}\right),\left(a_{2}, a_{1}\right),\left(a_{2}, a_{2}\right)\right\}$.

An action model is somewhat similar to an epistemic model: an atomic action represents a possible action that can be executed by agents, and the accessibility relation $\sim_{i}$ expresses the uncertainty of agent $i$ about which action has been executed. But instead of having a valuation function, an action model features a function pre that assigns a precondition to each atomic action. In order to be executable in an epistemic state $w$, an action's precondition must be satisfied in that state, which in our case means that if an agent wants to send message $\phi$, then the agent must know $\phi$. Fig. 4 shows three examples of action models derived from agent 1 announcing $p$ in $\mathcal{S}_{1}, \mathcal{S}_{2}$, and $\mathcal{S}_{3}$, respectively, of Fig. 2 . It is interesting to note that $A_{(1, p)}^{\mathcal{S}_{1}}$ and $A_{(1, p)}^{\mathcal{S}_{2}}$ are identical, while $A_{(1, p)}^{\mathcal{S}_{3}}$ is equivalent to a public announcement of $p$, since all other agents follow agent 1 .

We can now formally define entailment for $\mathcal{L}_{\text {SNL }}$ wrt. social epistemic models.

Definition 7 (Semantics). Given a social epistemic model $E=\langle A g, \mathcal{S}, M\rangle$ and a social epistemic formula $\phi \in \mathcal{L}_{\mathrm{SNL}}$, the entailment relation $\models$ is defined by

$$
\begin{array}{ll}
E, w \models p & \text { iff } w \in V(p) ; \\
E, w \models f_{(i, j)} & \text { iff } i F_{\mathcal{S}} j ; \\
E, w \models \neg \phi & \text { iff } E, w \models \phi ; \\
E, w \models \phi \wedge \psi & \text { iff } E, w \models \phi \text { and } E, w \models \psi ; \\
E, w \models K_{i} \phi & \text { iff for all } w^{\prime}, \text { if } w \sim_{i} w^{\prime} \text { then } E, w^{\prime} \models \phi ; \\
E, w \models C_{G} \phi & \text { iff for all } w^{\prime} \text {, if } w \sim_{G} w^{\prime} \text { then } E, w^{\prime} \models \phi ; \\
E, w \models\langle i: \operatorname{Fo}(j)\rangle \phi & \text { iff } E^{\prime}, w \models \phi \text { where } E^{\prime}=\left\langle A g, \mathcal{S}^{\prime}, M\right\rangle \text { and } \\
& F_{\mathcal{S}^{\prime}=F_{\mathcal{S}} \cup\{(i, j)\} ;} \\
E, w \models\langle i: \mathrm{uFo}(j)\rangle \phi & \text { iff } E^{\prime}, w \models \phi \text { where } E^{\prime}=\left\langle A g, \mathcal{S}^{\prime}, M\right\rangle \text { and } \\
& F_{\mathcal{S}^{\prime}=F_{\mathcal{S}} \backslash\{(i, j)\} ;} \\
E, w \models\langle i: \phi\rangle \psi & \text { iff } E_{, w \models}=K_{i} \phi \text { and } E^{\prime},\left(w, a_{1}\right) \models \psi \text { where } \\
& E^{\prime}=\langle A g, \mathcal{S}, M\rangle \otimes A_{(i, \phi)}^{\mathcal{S}} .
\end{array}
$$

The update operation $\otimes$ is given as $\langle A g, \mathcal{S}, M\rangle \otimes A_{(i, \phi)}^{\mathcal{S}} \equiv\left\langle A g, \mathcal{S}, M^{\prime}\right\rangle$, where $W_{M^{\prime}}=\left\{(w, a) \mid M, w \models \operatorname{pre}(a), a \in\left\{a_{1}, a_{2}\right\}\right\} ;(w, a) \sim_{i}\left(w^{\prime}, a^{\prime}\right)$ iff $w \sim_{i} w^{\prime}$ and $a \sim_{i} a^{\prime}$; and $(w, a) \in V_{M^{\prime}}(p)$ iff $w \in V_{M}(p)$. 


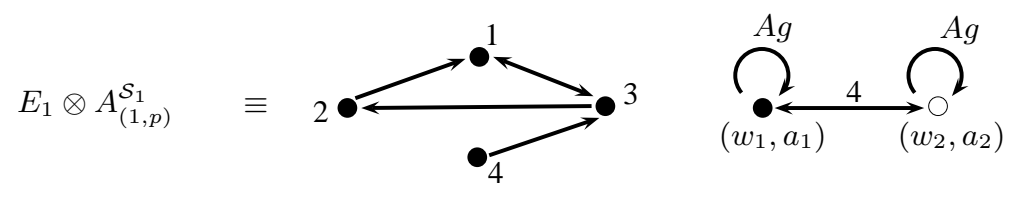

Fig. 5. Example Update Result.

The definition of the update operation $\otimes$ essentially follows from [3]. The main difference is that the action models in our approach are constructed based on social networks. Intuitively $\otimes$ takes a social epistemic model and an action mode to produce a new social epistemic model. Agent $i$ cannot distinguish two new worlds $(w, a)$ and $\left(w^{\prime}, a^{\prime}\right)$ if $i$ cannot distinguish the world $w$ from $w^{\prime}$ nor the action $a$ from $a^{\prime}$.

The other logical connectives $\vee, \rightarrow$ can be defined as usual. If $E, w \models \phi$ for all $E, w$, then $\phi$ is valid, written as $\models \phi$. To illustrate the update operation, Fig. 5 gives the result of updating $E_{1}$ from Fig. 3 with $A_{(1, p)}^{\mathcal{S}_{1}}$ from Fig. 4 . In the resulting model, the social network remains the same and $p$ becomes common knowledge for agents $1,2,3$, but agent 4 is still ignorant about $p$. Formally,

$$
E_{1}, w_{1} \models\langle 1: p\rangle\left(C_{\{1,2,3\}} p \wedge \neg K_{4} p\right) .
$$

Example: Revolt or Stay-at-home. Our new Social Network Logic provides a framework for formally analysing the motivating example from the introduction. Let $p_{(A, x)}$ represent the proposition "Agent $A$ (lice) will revolt if at least $x$ of her friends intend to revolt." It suffices to consider $x \in\{0,1,2,3,4\}$, where $x=0$ means $A$ will definitely revolt and $x=4$ means $A$ will definitely stay at home, given that there are only three other agents in this example. Each possible collection of the thresholds for every agent constitutes a possible world. We describe a world by tuples like 0123, which means that agent $A$ 's threshold is $0, B$ 's is $1, C$ 's is 2 , and $D$ 's is 3 . There are $5^{4}=625$ different possible worlds in total. Initially, each agent only knows about his or her own threshold. The accessibility relation for agent $A$ is defined as $\left(i j k l \sim_{A} i^{\prime} j^{\prime} k^{\prime} l^{\prime}\right)$ iff $i=i^{\prime}$, for agent $B$ as $\left(i j k l \sim_{B} i^{\prime} j^{\prime} k^{\prime} l^{\prime}\right)$ iff $j=j^{\prime}$, and similar for agents $C$ and $D$. Valuation $V$ is defined naturally as $V\left(p_{(A, x)}\right) \equiv\{i j k l \mid i=x\}$ and similar for the other atomic propositions. Let $E_{\text {init }}^{L}$ denote the initial social epistemic model with the network on the left-hand side of Fig. $1 ; E_{\text {init }}^{R}$ the initial social epistemic model with the network on the right-hand side. We use $E_{\text {init }}$ to refer to either of them. Assume that our agents all have threshold 2 (i.e., world 2222) and only know their own threshold initially. The following (about everyone's knowledge of agent $A$ 's threshold) can be formally concluded for both social network structures:

$$
E_{\text {init }}, 2222 \models K_{A} p_{(A, 2)} \wedge \neg K_{B} p_{(A, 2)} \wedge \neg K_{C} p_{(A, 2)} \wedge \neg K_{D} p_{(A, 2)} .
$$

After $A$ posts her threshold, what agents know about $p_{(A, 2)}$ starts to diverge:

$$
E_{\text {init }}^{L}, 2222 \models\left\langle A: p_{(A, 2)}\right\rangle\left(K_{A} p_{(A, 2)} \wedge K_{B} p_{(A, 2)} \wedge \neg K_{C} p_{(A, 2)} \wedge K_{D} p_{(A, 2)}\right),
$$




$$
E_{\text {init }}^{R}, 2222 \models\left\langle A: p_{(A, 2)}\right\rangle\left(K_{A} p_{(A, 2)} \wedge K_{B} p_{(A, 2)} \wedge K_{C} p_{(A, 2)} \wedge K_{D} p_{(A, 2)}\right) .
$$

After all agents have posted their thresholds, in the epistemic model resulting from $\left(E_{\text {init }}^{L}, 2222\right)$, agent $A$ knows that $C$ considers it possible that $p_{(A, 4)}$ is true:

$$
E_{\text {init }}^{L}, 2222 \models\left\langle A: p_{(A, 2)}\right\rangle\left\langle B: p_{(B, 2)}\right\rangle\left\langle C: p_{(C, 2)}\right\rangle\left\langle D: p_{(D, 2)}\right\rangle\left(K_{A} \neg K_{C} \neg p_{(A, 4)}\right) .
$$

Hence, agent $C$ considers it possible that $A$ will stay at home. A similar analysis applies to $B$ 's ignorance about $D$ 's threshold. So from $C$ 's perspective, agent $B$ may think it is possible that both $A$ and $D$ will stay at home. Therefore, $A$ will also choose to stay at home. The key condition for them to revolt, namely, that they should have common knowledge about their thresholds among a group of at least three, is not given. Formally, $\left(E_{\text {init }}^{L}, 2222\right)$ does not entail

$$
\left\langle A: p_{(A, 2)}\right\rangle\left\langle B: p_{(B, 2)}\right\rangle\left\langle C: p_{(C, 2)}\right\rangle\left\langle D: p_{(D, 2)}\right\rangle\left(C_{\{A, B, C\}}\left(p_{(A, 2)} \wedge p_{(B, 2)} \wedge p_{(C, 2)}\right)\right) \text {. }
$$

The situation is different for $\left(E_{\text {init }}^{R}, 2222\right)$, where agents $A, B, C$ manage to achieve common knowledge about their thresholds. Formally, $\left(E_{\text {init }}^{R}, 2222\right)$ entails

$$
\left\langle A: p_{(A, 2)}\right\rangle\left\langle B: p_{(B, 2)}\right\rangle\left\langle C: p_{(C, 2)}\right\rangle\left\langle D: p_{(D, 2)}\right\rangle\left(C_{\{A, B, C\}}\left(p_{(A, 2)} \wedge p_{(B, 2)} \wedge p_{(C, 2)}\right)\right) .
$$

Here, agents $A, B, C$ will all revolt while agent $D$ chooses to stay at home.

\section{Theoretical Results}

Having illustrated how our new logic can be used to formally analyse the flow of knowledge in a social network, we now present some general results. First, we will formally prove that the existing PAL can be obtained as a special case of our SNL by introducing a special agent who knows everything, and a social network in which every agent follows this special agent.

Proposition 1. Given an epistemic model $M=\left\langle W,\left\{\sim_{i}: i \in A g\right\}, V\right\rangle$, there is a corresponding social epistemic model $E$ s.t. for all public announcements of $\phi$,

$$
M, w \models\langle\phi\rangle \psi \quad \text { iff } \quad E, w \models\langle\text { Announcer }: \phi\rangle \psi
$$

where Announcer is a special agent in $E$ and $\phi, \psi$ are formulas in $\mathcal{L}_{\mathrm{PAL}}$.

Proof. We construct $E=\left\langle A g^{\prime}, \mathcal{S}, M^{\prime}\right\rangle$ from $M$ as follows:

- $A g^{\prime}=A g \cup\{$ Announcer $\}$; special atoms $f_{(i, j)}$ are introduced for $i, j \in A g^{\prime}$;

- $\mathcal{S}=\left\langle A g^{\prime}, F\right\rangle$ with $(i$, Announcer $) \in F$ for all $i \in A g$;

- $M^{\prime}$ is exactly the same as $M$ except that $\sim_{\text {Announcer }}=\{(w, w) \mid w \in W\}$.

From left to right: Assume $M, w \models\langle\phi\rangle \psi$, then $M, w \models \phi$ and $M \mid \phi, w \models \psi$. Since $\sim_{\text {Announcer }}$ is the identity relation, we have $E, w \models K_{\text {Announcer }} \phi$. Action model $A_{(\text {Announcer }, \phi)}^{\mathcal{S}}$ has only identity pairs since all agents in $A g$ follow Announcer (the model is similar to $A_{(1, p)}^{\mathcal{S}_{3}}$ in Fig. 4). It is easy to show that $M \mid \phi$ is isomorphic to the epistemic part of $E \otimes A_{(\text {Announcer, } \phi)}^{\mathcal{S}}$. By structural induction on $\psi$, we have $E \otimes A_{(\text {Announcer, } \phi)}^{\mathcal{S}},\left(w, a_{1}\right) \models \psi$. Therefore $E, w \models\langle$ Announcer $: \phi\rangle \psi$ holds as desired. Since this line of argument is reversible, we immediately have the other direction. 
The above result concerns model equivalence, but we can also show equivalence of formula validity.

Proposition 2. Let a syntactic translation trs from $\mathcal{L}_{\mathrm{PAL}}$ to $\mathcal{L}_{\mathrm{SNL}}$ be given as:

$$
\begin{array}{rlrl}
\operatorname{trs}(p) & \equiv p & \operatorname{trs}\left(K_{i} \phi\right) \equiv K_{i} \operatorname{trs}(\phi) \\
\operatorname{trs}(\phi \wedge \psi) \equiv \operatorname{trs}(\phi) \wedge \operatorname{trs}(\psi) & & \operatorname{trs}\left(C_{G} \phi\right) \equiv C_{G} \operatorname{trs}(\phi) \\
\operatorname{trs}(\neg \phi) & \equiv \neg \operatorname{trs}(\phi) & \operatorname{trs}(\langle\phi\rangle \psi) \equiv\langle\text { Announcer }: \operatorname{trs}(\phi)\rangle \operatorname{trs}(\psi) \\
\text { then } \models_{\mathrm{PAL}} \phi \text { iff } \models \mathrm{SNL} \bigwedge_{i \in A g} f_{(i, \text { Announcer })} \rightarrow \operatorname{trs}(\phi) .
\end{array}
$$

Proof. Assume $\models_{\mathrm{PAL}} \phi$. Take an arbitrary social epistemic model $E=\langle A g \cup$ $\{$ Announcer $\}, \mathcal{S}, M\rangle$ and a world $w$. Assume $E, w \models \mathrm{SNL} \bigwedge_{i \in A g} f_{(i, \text { Announcer })}$. $\mathcal{S}$ is a social network where every agent follows the Announcer, and $M, w \models$ PAL $\phi$ follows from the assumption. By induction on $\phi$ and using similar reasoning as in Proposition $1, E, w \models_{\mathrm{SNL}} \operatorname{trs}(\phi)$. Similar for the other direction.

Our next results are about whether two actions can be executed in a different order and still result in epistemic situations that satisfy the same formulas, that is, $\models\left\langle\pi_{1}\right\rangle\left\langle\pi_{2}\right\rangle \phi \leftrightarrow\left\langle\pi_{2}\right\rangle\left\langle\pi_{1}\right\rangle \phi$. Clearly, this will depend on the type of actions. For the network actions alone, if $\pi_{1}, \pi_{2}$ are both of the type of $i: \operatorname{Fo}(j)$ or $i: \operatorname{uFo}(j)$, then the above principle indeed holds: It is easy to verify, for instance, that

$$
\models\langle i: \operatorname{Fo}(j)\rangle\langle k: \operatorname{Fo}(l)\rangle \phi \leftrightarrow\langle k: \operatorname{Fo}(l)\rangle\langle i: \operatorname{Fo}(j)\rangle \phi .
$$

But clearly we cannot in general mix different structural actions, that is,

$$
\not=\langle i: \operatorname{Fo}(j)\rangle\langle i: \mathrm{uFo}(j)\rangle \phi \leftrightarrow\langle i: \mathrm{uFo}(j)\rangle\langle i: \operatorname{Fo}(j)\rangle \phi
$$

More interesting are cases which also involve message actions:

$$
\forall=\langle j: \phi\rangle\langle i: \mathrm{uFo}(j)\rangle \psi \leftrightarrow\langle i: \mathrm{uFo}(j)\rangle\langle j: \phi\rangle \psi .
$$

However, for a special class of models where $i$ does not follow $j$, we do have

$$
\models \neg f_{(i, j)} \rightarrow(\langle j: \phi\rangle\langle i: \mathrm{uFo}(j)\rangle \psi \leftrightarrow\langle i: \mathrm{uFo}(j)\rangle\langle j: \phi\rangle \psi) .
$$

The next result says that if two agents know some propositional facts initially, then no matter in what order they post these facts, the resulting models will satisfy the same formulas (i.e., no formulas can distinguish them).

Proposition 3. Given propositional formulas $\phi_{1}, \phi_{2}$,

$$
\models K_{i} \phi_{1} \wedge K_{j} \phi_{2} \rightarrow\left(\left\langle i: \phi_{1}\right\rangle\left\langle j: \phi_{2}\right\rangle \psi \leftrightarrow\left\langle j: \phi_{2}\right\rangle\left\langle i: \phi_{1}\right\rangle \psi\right) .
$$

Proof. Given an arbitrary epistemic model $E$ and a world $w$, assume $E, w \models$ $K_{i} \phi_{1} \wedge K_{j} \phi_{2}$. From left to right: Assume $E, w \models\left\langle i: \phi_{1}\right\rangle\left\langle j: \phi_{2}\right\rangle \psi$. It follows that $E, w \models K_{i} \phi_{1}$ and $E \otimes A_{\left(i, \phi_{1}\right)},\left(w, a_{1}\right) \models\left\langle j: \phi_{2}\right\rangle \psi$; hence, $E \otimes A_{\left(i, \phi_{1}\right)},\left(w, a_{1}\right) \models$ $K_{j} \phi_{2}$ and $E \otimes A_{\left(i, \phi_{1}\right)} \otimes A_{\left(j, \phi_{2}\right)},\left(\left(w, a_{1}\right), a_{1}\right) \models \psi$. There is an isomorphism between $E \otimes A_{\left(i, \phi_{1}\right)} \otimes A_{\left(j, \phi_{2}\right)}$ and $E \otimes A_{\left(j, \phi_{2}\right)} \otimes A_{\left(i, \phi_{1}\right)}$ by mapping every $((w, a), b)$ to $((w, b), a)$. It is easy to show that $E \otimes A_{\left(j, \phi_{2}\right)} \otimes A_{\left(i, \phi_{1}\right)},\left(\left(w, a_{1}\right), a_{1}\right) \models \psi$, and $E \otimes A_{\left(j, \phi_{2}\right)},\left(w, a_{1}\right) \models K_{i} \phi_{1}$ (as $E, w \models K_{i} \phi_{1}$ and $\phi_{1}, \phi_{2}$ are propositional). We then have $E, w \models\left\langle j: \phi_{2}\right\rangle\left\langle i: \phi_{1}\right\rangle \psi$. The other direction is similar. 
It is worth noting that this may not hold if we lift the restriction on $\phi_{1}, \phi_{2}$ to be propositional (i.e., without modal operator). Suppose, for example, a situation $E, w$ where $j F i$ and $h F i$ along with $K_{i} p$ and $K_{j} \neg K_{h} p$ hold. We then have $E, w \models\left\langle j: \neg K_{h} p\right\rangle\langle i: p\rangle \top$, but not $E, w \models\langle i: p\rangle\left\langle j: \neg K_{h} p\right\rangle \top$.

Finally, we consider the question whether two message actions have the same effect as one. Again this depends on the social network. While it is not true in general it does hold under certain conditions. Let us consider one result for transitive social networks, i.e., where $\bigwedge_{i, j, h \in A g}\left(f_{(i, j)} \wedge f_{(j, h)} \rightarrow f_{(i, h)}\right)$.

Proposition 4. Given propositional formula $\phi$ and two agents $m, n \in A g$ :

$$
\models f_{(m, n)} \wedge \bigwedge_{i, j, h \in A g}\left(f_{(i, j)} \wedge f_{(j, h)} \rightarrow f_{(i, h)}\right) \rightarrow(\langle n: \phi\rangle \psi \leftrightarrow\langle n: \phi\rangle\langle m: \phi\rangle \psi) .
$$

Proof. Given a social epistemic model $E=\langle A g, \mathcal{S}, M\rangle$, assume $E, w \models f_{(m, n)} \wedge$ $\bigwedge_{i, j, h \in A g}\left(f_{(i, j)} \wedge f_{(j, h)} \rightarrow f_{(i, h)}\right)$. This ensures that $\mathcal{S}$ is transitive and $m F n$. From left to right: Suppose $E, w \models\langle n: \phi\rangle \psi$. So it is the case that $E, w \models K_{n} \phi$ and $E \otimes A_{(n, \phi)},\left(w, a_{1}\right) \models \psi$. From transitivity and $m F n$, we know that $\{h \mid$ $h F m\} \subseteq\{h \mid h F n\}$, that is, all followers of $m$ are also followers of $n$. Since $\phi$ is propositional, we can show that $E \otimes A_{(n, \phi)}$ and $E \otimes A_{(n, \phi)} \otimes A_{(m, \phi)}$ are isomorphic. It follows that $E \otimes A_{(n, \phi)} \otimes A_{(m, \phi)},\left(\left(w, a_{1}\right), a_{1}\right) \models \psi$, and hence $E, w \models\langle n: \phi\rangle\langle m: \phi\rangle \psi$. The other direction follows by reverse reasoning.

\section{Conclusion}

We have introduced a logic for reasoning about knowledge and change in social networks that generalises Public Announcement Logic by an information flow network. For further research, we intend to find an axiomatic system to characterise communications in social networks, and to study the computational complexity of verifying formulas for given social epistemic models.

We conclude with a short discussion of related work. Ruan [12] gives a logic of private message passing in which a message expression $C C_{G} \phi$ denotes a private message $\phi$ being sent to group $G$ (similar to email Carbon Copying). Roelofsen [11] proposes a more general logic by introducing the notion of communication channels among groups $G_{1}$ and $G_{2}$, and message actions by which group $G_{1}$ sends a message $\phi$ to group $G_{2}$. Seligman et al. [13] propose a Facebook Logic that has an explicit social network as part of a possible world and where the social relations are assumed to be symmetric. All of these frameworks use a dynamic epistemic semantics based on [3] or [4].

Pacuit and Parikh [10] give a logic of communication graphs by introducing a commonly known, static, directed graph, which explicitly represents the communication links between individual agents, and a temporal expression $\diamond \phi$, which represents that "after some communications, $\phi$ becomes true". They use a history-based semantics rather than dynamic epistemic semantics of $[3,4]$. Apt et al. [2] and Wang et al. [15] also use a history-based semantics, but the communication structure (named hypergraph) is formed on groups: A message from an 
agent is received by all members of the same group. Both [10] and [2] limit the message contents to atomic propositions, and message actions are specified only in the history model, while [15] has a richer language to represent both message contents and actions. Sietsma and van Eijck in their recent paper [14] propose a framework for message passing that combines the dynamic epistemic semantics and history-based approaches.

Using a dynamic epistemic semantics, our work is in line with $[12,11,13]$. Different from $[12,11]$, our communication channels are explicitly represented in social networks, and we link individual agents rather than groups. Different from [13], our social network does not need to be symmetric and is not part of a possible world; in addition, we have actions that changes network structures. Our work shares with [10] the assumption that the social network relations are commonly known by the agents. This assumption might be too strong for reallife social networks in which the social relationships between agents are quite complex and highly context-dependent. However, we can generalise our approach in a similar style as [13] by making a social network as part of a possible world.

\section{References}

1. Abraham, A., Hassanien, A.E., Snasel, V. (eds.): Computational Social Network Analysis. Springer-Verlag London (2010)

2. Apt, K.R., Witzel, A., Zvesper, J.A.: Common knowledge in interaction structures. In: Heifetz, A. (ed.) Proc. of TARK. pp. 4-13 (2009)

3. Baltag, A., Moss, L.: Logics for epistemic programs. Synthese 139, 165-224 (2004), knowledge, Rationality \& Action 1-60

4. van Benthem, J., van Eijck, J., Kooi, B.P.: Logics of communication and change. Inf. Comput. 204(11), 1620-1662 (2006)

5. Chwe, M.S.Y.: Structure and strategy in collective action. American Journal of Sociology 105(1), 128-156 (1999)

6. van Ditmarsch, H., van der Hoek, W., Kooi, B.: Dynamic Epistemic Logic, Synthese Library, vol. 337. Springer (2007)

7. Fagin, R., Halpern, J.Y., Moses, Y., Vardi, M.Y.: Reasoning About Knowledge. MIT Press (1995)

8. Freeman, L.: The Development of Social Network Analysis: A Study in the Sociology of Science. Empirical Press, Vancouver Canada (2004)

9. Jackson, M.: Social and Economic Networks. Princeton University Press (2008)

10. Pacuit, E., Parikh, R.: Reasoning about communication graphs. In: van Benthem, J., Loewe, B., Gabbay, D. (ed.) Interactive Logic: Games and Social Software (2007)

11. Roelofsen, F.: Exploring Logical Perspectives on Distributed Information and its Dynamics. Master's thesis, ILLC, University of Amsterdam (2005)

12. Ruan, J.: Exploring the Update Universe. Master's thesis, ILLC Publications, Master of Logic Thesis Series, University of Amsterdam (2004)

13. Seligman, J., Liu, F., Girard, P.: Logic in the community. In: Banerjee, M., Seth, A. (eds.) Proc. of ICLA. LNCS, vol. 6521, pp. 178-188. Springer (2011)

14. Sietsma, F., van Eijck, J.: Message passing in a dynamic epistemic logic setting. In: Apt, K.R. (ed.) Proc. of TARK. pp. 212-220. ACM (2011)

15. Wang, Y., Sietsma, F., van Eijck, J.: Logic of information flow on communication channels. In: Omicini, A., Sardina, S., Vasconcelos, W.W. (eds.) Proc. of DALT. LNCS, vol. 6619, pp. 130-147. Springer (2010) 